Proyecciones Journal of Mathematics

Vol. 28, No 2, pp. 125-132, August 2009.

Universidad Católica del Norte

Antofagasta - Chile

\title{
ON EXISTENCE OF PERIODIC SOLUTION TO CERTAIN NONLINEAR THIRD ORDER DIFFERENTIAL EQUATIONS
}

\author{
CEMIL TUNÇ \\ Yüzüncü Yil University, Turkey \\ Received : October 2008. Accepted : March 2009
}

\begin{abstract}
In this paper, it is investigated the existence of periodic solutions to the nonlinear third order differential equation:

$$
x^{\prime \prime \prime}+c_{2}(t) x^{\prime \prime}+c_{1}(t) x^{\prime}+f(t, x)=p\left(t, x, x^{\prime}, x^{\prime \prime}\right) .
$$

The Leray-Schauder principle is used to show the existence of periodic solutions of this equation.
\end{abstract}

Subjclass : [AMS] 34C25, 65L06.

Keywords : Periodic solution, nonlinear third order differential equation. 


\section{Introduction}

With respect to our observations, up to now, the problem of existence of periodic solutions for various nonlinear second and third order differential equations have been studied in the literature by only a few authors, see, for example, the papers of Mehri \& Shadman [1], Shadman \& Mehri [2] and Tunç \& Çinar [3].

Meanwhile, it is also worth mentioning that, in 1995, Shadman \& Mehri [2] discussed the existence of periodic solutions to nonlinear third order differential equation of the form:

$$
x^{\prime \prime \prime}+c_{2}(t) x^{\prime \prime}+c_{1}(t) x^{\prime}+f(t, x)=e(t) .
$$

In [2], the authors used Leray-Schauder principle to show the existence of periodic solutions of this equation.

In this paper, we discuss the same topic as in ([1], [2], [3]) to nonlinear third order differential equation of the type:

$$
x^{\prime \prime \prime}+c_{2}(t) x^{\prime \prime}+c_{1}(t) x^{\prime}+f(t, x)=p\left(t, x, x^{\prime}, x^{\prime \prime}\right),
$$

in which $p\left(t, x, x^{\prime}, x^{\prime \prime}\right), f(t, x), c_{1}(t)$ and $c_{2}(t)$ are continuous functions in their respective domains $[0, \omega) \times R^{3},[0, \omega) \times R$ and $[0, \omega)$, respectively. In addition, it is assumed that all initial value problems corresponding to equation $(1.1)$ can be extended to $[0, \omega)$. Clearly, our equation, $(1.1)$, includes the equation investigated by Shadman and Mehri [2].

\section{Main Result}

Our main result is the following theorem.

Theorem 2.1. In addition to the basic assumptions imposed on the functions $c_{1}, c_{2}, f$ and $p$ that appearing in equation (1.1), we assume that the following conditions hold:

i) $|f(t, x)| \leq \gamma|x|+\beta$, forallt $\in[0, \omega]$ and $|x|<\infty$ where $\gamma$ and $\beta$ are some non-negative constants.

(ii) $\left|p\left(t, x, x^{\prime}, x^{\prime \prime}\right)\right| \leq|e(t)|$, for all $t, x, x^{\prime}$ and $x^{\prime \prime}$, and $|e(t)|$ is a continuous function for all $t \in[0, \omega]$.

$[0, \omega]$

iii) $\left(\frac{\omega}{\pi}\right)^{3}+\gamma_{1}\left(\frac{\omega}{\pi}\right)^{2}+\gamma_{2}\left(\frac{\omega}{\pi}\right)<1, \gamma_{i}=\max \left|c_{i}(t)\right|, \quad(i=1,2)$ and $t \in$ 
Then equation (1.1) possesses a solution satisfying the boundary conditions,

$$
x^{(i)}(0)+x^{(i)}(\omega)=0,(i=0,1,2) .
$$

Proof. First, we show an estimate on the magnitude of the solutions of problem:

$$
\begin{gathered}
x^{\prime \prime \prime}+c_{2}(t) x^{\prime \prime}+c_{1}(t) x^{\prime}=\mu\left[p\left(t, x, x^{\prime}, x^{\prime \prime}\right)-f(t, x)\right], \mu \in[0,1], \\
x^{(i)}(0)+x^{(i)}(\omega)=0,(i=0,1,2) .
\end{gathered}
$$

It is assumed that $x(t)$ is a function of class $C^{n-1}[0, \omega]$ such that $x(t+$ $\omega)+x(t)=0$ for all $t$, and we also use Wirtinger's inequalities in the following from:

$$
\left\|x^{(i-1)}(t)\right\|_{2} \leq\left(\frac{\omega}{\pi}\right)^{n-i+1}\left\|x^{(n)}(t)\right\|_{2},(i=1,2, \ldots, n),
$$

where

$$
\|\cdot\|_{2}=\left[\int_{0}^{\omega}|\cdot|^{2} d t\right]^{\frac{1}{2}}
$$

Now, we suppose that $x(t)$ is a solution of the problem given by (2.2). In the light of the assumptions of the theorem, we have from (2.2) that;

$$
\begin{gathered}
\left|x^{\prime \prime \prime}(t)\right| \leq\left|c_{2}(t)\right|\left|x^{\prime \prime}(t)\right|+\left|c_{1}(t)\right|\left|x^{\prime}(t)\right|+\mu\left[\left|p\left(t, x(t), x^{\prime}(t), x^{\prime \prime}(t)\right)\right|+|f(t, x)|\right] \\
\leq \gamma_{2}\left|x^{\prime \prime}(t)\right|+\gamma_{1}\left|x^{\prime}(t)\right|+\mu[|e(t)|+\gamma|x(t)|+\beta] .
\end{gathered}
$$

Hence, by using the Minkowski's inequality, we get

$$
\left\|x^{\prime \prime \prime}(t)\right\|_{2} \leq \gamma_{2}\left\|x^{\prime \prime}(t)\right\|_{2}+\gamma_{1}\left\|x^{\prime}(t)\right\|_{2}+\mu\left\{\|e(t)\|_{2}+\gamma\|x(t)\|_{2}+\beta \sqrt{\omega}\right\} \text {. }
$$

It can also be seen from Wirtinger's inequality (2.3) that 


$$
\begin{aligned}
& \left\|x^{\prime \prime \prime}(t)\right\|_{2} \leq\left\{\gamma_{2}\left(\frac{\omega}{\pi}\right)+\gamma_{1}\left(\frac{\omega}{\pi}\right)^{2}\right\}\left\|x^{\prime \prime \prime}(t)\right\|_{2} \\
& +\mu\left\{\|e(t)\|_{2}+\gamma\left(\frac{\omega}{\pi}\right)^{3}\left\|x^{\prime \prime \prime}(t)\right\|_{2}+\beta \sqrt{\omega}\right\} .
\end{aligned}
$$

Hence,

$$
\left[1-\gamma_{2}\left(\frac{\omega}{\pi}\right)-\gamma_{1}\left(\frac{\omega}{\pi}\right)^{2}-\gamma \mu\left(\frac{\omega}{\pi}\right)^{3}\right]\left\|x^{\prime \prime \prime}(t)\right\|_{2} \leq \mu\left\{\|e(t)\|_{2}+\beta \sqrt{\omega}\right\} .
$$

On the other hand, since

$$
0<\gamma \mu\left(\frac{\omega}{\pi}\right)^{3}+\gamma_{1}\left(\frac{\omega}{\pi}\right)^{2}+\gamma_{2}\left(\frac{\omega}{\pi}\right)<1
$$

then, clearly, it follows

$$
\left\|x^{\prime \prime \prime}(t)\right\|_{2} \leq \frac{\mu\left\{\|e(t)\|_{2}+\beta \sqrt{\omega}\right\}}{1-\gamma_{2}\left(\frac{\omega}{\pi}\right)-\gamma_{1}\left(\frac{\omega}{\pi}\right)^{2}-\gamma \mu\left(\frac{\omega}{\pi}\right)^{3}} .
$$

Now, let

$$
\Delta_{0}=\frac{\left\{\|e(t)\|_{2}+\beta \sqrt{\omega}\right\}}{1-\gamma_{2}\left(\frac{\omega}{\pi}\right)-\gamma_{1}\left(\frac{\omega}{\pi}\right)^{2}-\gamma \mu\left(\frac{\omega}{\pi}\right)^{3}} .
$$

Thus,

$$
\left\|x^{\prime \prime \prime}(t)\right\|_{2} \leq \mu \Delta_{0}
$$

Next, we write

$$
x^{(i-1)}(t)=x^{(i-1)}(0)+\int_{0}^{t} x^{(i)}(\tau) d \tau, \quad(i=1,2,3) .
$$

Let us take $t=\omega$. Then, we have 


$$
x^{(i-1)}(\omega)=x^{(i-1)}(0)+\int_{0}^{\omega} x^{(i)}(\tau) d \tau,(i=1,2,3) .
$$

In view of (2.5) and (2.1), it follows that

$$
0=2 x^{(i-1)}(0)+\int_{0}^{\omega} x^{(i)}(\tau) d \tau,(i=1,2,3),
$$

and we also get

$$
x^{(i-1)}(0)=-x^{(i-1)}(\omega)=-\frac{1}{2} \int_{0}^{\omega} x^{(i)}(\tau) d \tau, \quad(i=1,2,3) .
$$

Hence,

$$
x^{(i-1)}(t)=-\frac{1}{2} \int_{0}^{\omega} x^{(i)}(\tau) d \tau+\int_{0}^{t} x^{(i)}(\tau) d \tau=\frac{1}{2} \int_{0}^{t} x^{(i)}(\tau) d \tau, \quad(i=1,2,3) .
$$

Clearly, the last equality yields that

$$
\left|x^{(i-1)}(t)\right| \leq \frac{1}{2} \int_{0}^{\omega}\left|x^{(i)}(\tau)\right| d \tau, \quad(i=1,2,3) .
$$

Now, obviously, it follows from (2.6) that

$$
\left|x^{(i-1)}(t)\right| \leq \frac{1}{2} \sqrt{\omega}\left\|x^{(i)}(t)\right\|_{2}, \quad(i=1,2,3) .
$$

Combining the inequality (2.7) with Wirtinger's inequality and (2.4), we obtain

$$
\left|x^{(i-1)}(t)\right| \leq \frac{\mu}{2} \sqrt{\omega}\left(\frac{\omega}{\pi}\right)^{4-i} \Delta_{0}, 0 \leq \mu \leq 1, \quad(i=1,2,3) .
$$

For $\mu=0$, it is clear from (2.8) that

$$
x^{(i-1)}(t)=0, t \in[0, \omega], \quad(i=1,2,3) .
$$

That is, $x(t)=0, x^{\prime}(t)=0$ and $x^{\prime \prime}(t)=0$. In this case, it follows that the homogenous equation 


$$
x^{\prime \prime \prime}+c_{2}(t) x^{\prime \prime}+c_{1}(t) x^{\prime}=0
$$

has no non-trivial solution which satisfies the boundary conditions (2.1),

$$
x^{(i)}(0)+x^{(i)}(\omega)=0,(i=0,1,2) .
$$

This guarantees the existence of a Greens function for the problem (2.2). Clearly, the problem (2.2) is equivalent to the following:

$$
x(t)=\mu \int_{0}^{\omega} g(t, s)\left[p\left(s, x(s), x^{\prime}(s), x^{\prime \prime}(s)\right)-f(s, x(s))\right] d s .
$$

Now, we consider the space $C^{3}[0, \omega]$ normed by

$$
\|x\|_{C^{3}}=\max \left|x^{(i-1)}(t)\right|, t \in[0, \omega], \quad(i=1,2,3) .
$$

Let $\mathbf{B}_{\rho}$ be the space

$$
\mathbf{B}_{\rho}=\left\{x(t) \in C^{3}[a, b]:\|x\|_{C^{3}} \leq \rho\right\}
$$

where

$$
\rho=\max _{i}\left\{\frac{1}{2} \sqrt{\omega}\left(\frac{\omega}{\pi}\right)^{4-i} \Delta_{0}\right\},(i=1,2,3) .
$$

In view of the sphere

$$
\mathbf{S}_{R}=\left\{x(t) \in C^{3}[a, b]:\|x\|_{C^{3}}=R\right\}
$$

it follows for arbitrary $R>\rho$ that equation (2.9) has no solution on the sphere $\mathbf{S}_{R}$. Now, by noting Leary-Schauder principle and the complete continuity of the operator

$$
(L x)(t)=\mu \int_{0}^{\omega} g(t, s)\left[p\left(s, x(s), x^{\prime}(s), x^{\prime \prime}(s)\right)-f(s, x(s))\right] d s
$$

one can conclude that equation (2.9) has at least a solution in the open ball $\left\{x:\|x\|_{C^{3}}<R\right\}$. 
As a result, there exists a solution of equation (2.9) in the closed ball $\mathbf{B}_{\rho}$. Therefore, this fact implies that the problem (2.2) has, at the least, a solution for $\mu=1$.

Remark. When we take $p(t, x, y, z)=e(t)$ in equation (1.1), then the conditions of theorem reduce those of Shadman and Mehri [2, Theorem 1]. It is also clear that our result generalizes that of Shadman and Mehri [2, Theorem 1].

Corollary. In addition to the assumptions of theorem, we assume that the following conditions hold:

(iv) The functions $c_{1}(t)$ and $c_{2}(t)$ are $\omega$-periodic, that is, $c_{1}(t+\omega) \equiv$ $c_{1}(t)$ and $c_{2}(t+\omega) \equiv c_{2}(t)$.

$(\mathbf{v})$ The function $p(t, x, y, z)$ is $2 \omega$-periodic, that is, $p(t+\omega, x, y, z) \equiv$ $p(t, x, y, z)$ and in addition $p(t+\omega, x, y, z) \equiv-p(t, x, y, z)$.

(vi) The functions $f(t, x)$ is $\omega$-periodic, that is, $f(t+\omega, x) \equiv f(t, x)$ and also $f(t,-x) \equiv-f(t, x)$.

Then equation (1.1) has a $2 \omega$-periodic solution with zero mean.

Proof : Let $\bar{x}(t)$ be a $2 \omega$-periodic extension of solution $x(t)$ defined as follows:

$$
\bar{x}(t)= \begin{cases}x(t), & 0 \leq t \leq \omega \\ -x(t+\omega) & -\omega \leq t \leq 0 .\end{cases}
$$

Clearly, $\bar{x}(t) \in C^{2}[-\omega, \omega]$. Next, in the light of the above assumptions imposed on the functions $c_{1}(t), c_{2}(t), f(t, x)$ and $p(t, x, y, z)$, it can be easily shown that $\bar{x}(t)$ is a solution of equation (1.1) which satisfies the periodic boundary condition:

$$
\bar{x}^{(i)}(\omega)=\bar{x}^{(i)}(-\omega), \quad(i=0,1,2) .
$$

Subject to the fact

$$
\int_{0}^{2 \omega} \bar{x}(t) d t=\int_{0}^{\omega} \bar{x}(t) d t+\int_{\omega}^{2 \omega} \bar{x}(t) d t
$$

it follows that

$$
\int_{0}^{2 \omega} \bar{x}(t) d t=\int_{0}^{\omega} \bar{x}(t) d t+\int_{0}^{\omega} \bar{x}(t+\omega) d t=0 .
$$

This fact shows that the solution $\bar{x}(t)$ has a zero mean value. The proof of corollary is now complete. 


\section{References}

[1] B. Mehri \& D. Shadman, On The Existence of Periodic Solutions of a Certain Class of Second Order Nonlinear Differential Equation, Math. Inequal. Appl. 1(3), pp. 431-436, (1998).

[2] D. Shadman \& B. Mehri, On The Periodic Solutions of Certain Nonlinear Third Order Differential Equations, Z. Angew. Math. Mech. (75)(2), pp. 164-166, (1995).

[3] C. Tunç \& I. Çınar, On the existence of periodic solutions to nonlinear differential equations of second order, Differ. Uravn. Protsessy Upr. (Differential Equations and Control Processes), (3), pp. 1-6, (2008).

[4] C. Tunç, On the existence of periodic solutins to a certain fourth-order nonlinear differential equation. Ann. Differential Equations 25, 1, pp. 8-12 (2009).

\section{Cemil Tunç}

Department of Mathematics, Faculty of Arts and Sciences Yüzüncü Yil University 65080, Van

Turkey

e-mail : cemtunc@yahoo.com 